María José Serrano*

\title{
The Construction of Advertising Discourse by the Use of the Second-Person Object $T e$ and the Clitic Se
} La construcción del discurso publicitario a través de la variación del objeto de persona te y del clítico se

https://doi.org/10.1515/soprag-2018-0005

Abstract: The purpose of this study is to analyze the variation of the secondperson object clitic te ['you'] and the clitic se [lit. 'it'] in advertising discourse from a cognitive viewpoint. The main explanatory notion will be cognitive salience; both clitics, te and se, exhibit this to varying degrees. The secondperson te is more salient than the clitic se; therefore, the meanings conveyed by each in media discourse will be notably different. Results indicate that the distribution and usage of the clitics te vs. se constitutes a different strategy of persuasion in advertising discourse. In general terms, te is used when a special deictic stance is needed, such as indexing a second-person as a potential consumer of the product or idea, whereas se tends to appear in utterances that describe the general and objective benefits of the product or idea advertised, denoting a desubjectivizing stance. The sociosituational distribution of this variation according to the target consumers will be analyzed.

Keywords: advertisement, clitics, se, syntax, te, variation

Resumen: En esta investigación se estudiará la variación que existe en el uso de la segunda persona te y el clítico se en el discurso publicitario como opciones significativas encaminadas a construir un mensaje que atraiga la atención del potencial consumidor y consiga su objetivo de forma efectiva. La segunda persona del singular, tanto en sus formas de sujeto (tú) como de objeto (te, a ti) es la forma más frecuentemente usada en el discurso publicitario. No obstante, en numerosos anuncios se observa también el uso del clítico se, lo cual podría indicar que se trata de una forma cuyo significado puede ser muy útil como estrategia persuasiva en este tipo de discurso. Como punto de partida

*Corresponding author: María José Serrano, Universidad de La Laguna, Santa Cruz de Tenerife, Spain, E-mail: mjserran@ull.edu.es 
para el análisis del significado de esta variación se utilizará la prominencia cognitiva. Los distintos valores de prominencia de las dos personas gramaticales analizadas se concretan en distintos significados en el discurso publicitario. Se estudiará la distribución sociosituacional de las variantes en función de los tipos de consumidores a los que van dirigidos los anuncios.

Palabras clave: Clíticos, publicidad, se, sintaxis, te, variación

\section{Introducción}

Las opciones comunicativas que ofrece la variación morfosintáctica se pueden observar en todos los ámbitos de la comunicación humana. Las variantes y sus valores de significado constituyen recursos que proveen a la situación comunicativa de distintos matices discursivo-pragmáticos. Es sabido que la variación en este nivel conlleva diferencias significativas, básicamente debido a las propiedades cognitivas que subyacen a la elección (Croft y Cruse, 2004 Langacker 1991 Langacker 2009). Esto implica que las formas gramaticales conllevan la creación de un significado que puede ser utilizado por los hablantes con fines e intenciones concretos en distintas situaciones comunicativas. Este constituye una estrategia discursiva en la cual la cognición posee una función primordial (Romano y Porto, 2016, p. 7). Por lo tanto, la perspectiva cognitiva, que implica que todo cambio en la forma conlleva un cambio en su significado, se revela como ideal para estudiar las variantes morfosintácticas en la actualidad. Las construcciones gramaticales en el discurso proporcionan la creación de distintos significados (Goldberg 2005) y es en la interacción comunicativa donde mejor pueden observarse $\mathrm{y}$, donde, en definitiva, termina por moldearse la gramática (Ginzburg 2012, p. 5). Por lo tanto, el análisis de la variación sintáctica debe realizarse bajo la premisa de que la gramática es variable y de que esta variabilidad está sustentada en la cognición y debe ser explicada de acuerdo a ella (Aijón Oliva 2018 Aijón Oliva y Serrano, 2013 pp. 11-45; Serrano 2016 pp. 816-817).

En esta investigación se estudiará la variación que existe entre el uso de la segunda persona en función de objeto te y el del clítico se en el discurso publicitario como alternativas encaminadas a construir un mensaje que atraiga la atención del potencial consumidor y consiga su objetivo de forma efectiva. La segunda persona del singular, tanto en sus formas de sujeto (tú) como de objeto (te, $a$ ti) es la forma más frecuentemente usada en el discurso publicitario. No obstante, en este tipo de discurso se observa también la presencia del clítico se, lo cual podría indicar que se trata de una forma cuyo significado puede ser muy útil también como estrategia persuasiva en este tipo de texto (Serrano 2018). 
Ambas variantes coaparecen en los distintos mensajes publicitarios de forma recurrente y sistemática, lo cual permite la creación de distintos estilos comunicativos concretos basados, como se verá, en la propiedad cognitiva de la prominencia.

\section{La indexación de la referencia personal y el discurso publicitario}

\subsection{Los fundamentos cognitivos de la referencia personal}

El distinto significado que surge de las variantes morfosintácticas está fundamentado en los distintos niveles de prominencia cognitiva. Esta es una conceptualización relativa al grado de accesibilidad o de relevancia que tiene una unidad en el discurso (Croft y Cruse 2004 pp. 46-50; Langacker 2009 p. 112), por lo tanto, un elemento será prominente si se encuentra activado en el transcurso de la interacción y es fácilmente recuperable a través de otros elementos lingüísticos (Ariel 2001 Gundel, Hedberg y Zacharski, 1993). Esto implica que no es necesaria su mención explícita en un momento determinado del discurso, ya que su referente es recuperable o accesible a través de otros recursos discursivo-pragmáticos.

La prominencia cognitiva se concreta y matiza además en valores tales como la perceptibilidad, la autonomía y la accesibilidad (Aijón Oliva 2018, cap. 2). La perceptibilidad está relacionada con la animacidad y con el grado de individuación de un referente. De acuerdo a ello, la mayor perceptibilidad de un referente se asocia a su construcción como primera y segunda persona: "Yo como", "tú comes". La tercera persona es la menos perceptible de todas, precisamente porque conlleva menos animacidad y una menor individuación del referente; de hecho, las terceras personas pueden referirse a cosas o entes inanimados: La casa está vacía. La autonomía alude a las funciones sintácticas, el sujeto y las funciones de dativo son más autónomos que las funciones de acusativo. Por último, la accesibilidad se refiere al nivel de identificación que tienen para los participantes las unidades en el discurso. Por lo tanto, el referente de un elemento pronominal será prominente si es accesible o perceptible para el hablante y se puede recuperar fácilmente en un contexto determinado.

Como punto de partida, la primera persona es la más prominente por representar la plena coincidencia con el participante que habla ("Yo camino por la calle"; Yo= la persona que habla). La segunda persona será menos prominente que la primera, pero más que la tercera. Es posible por lo tanto 
establecer un continuum de prominencia de las dos variantes que serán estudiadas en esta investigación: el objeto de segunda persona te es más prominente que se. En el ejemplo “Te cuento mi vida” es fácilmente deducible un referente de segunda persona en función de objeto central. En cambio, en "Se cuenta que vive aislado", no es posible recuperar ningún referente y la cláusula se convierte en impersonal. El clítico se tiene como referente a la tercera persona en sus usos reflexivos ("Él se peina a sí mismo), pero no recupera ningún referente cuando aparece en estructuras de pasiva refleja ("Se venden pisos") ni en las propiamente impersonales ("Se vive bien en Madrid").

Los referentes de te y se no solo son distintos, sino que entre ellos se puede establecer una gradualidad en su prominencia. La segunda persona te es más prominente que el clítico se, lo cual implica que la identificación de su referente o su accesibilidad o recuperación en el discurso es mucho más clara que la de se (Figura 1).

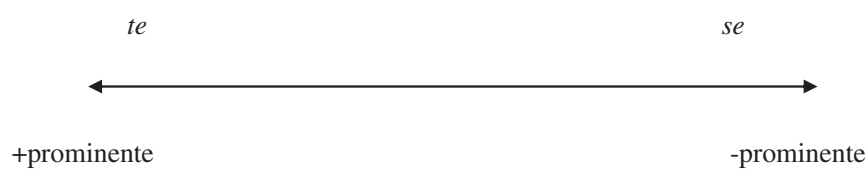

Figura 1: Continuum de prominencia de las variantes te y se.

Te y se son clíticos verbales o morfemas de concordancia (Anderson 2005 p. 240; Haspelmath 2013 Miller y Monachesi, 2003) los cuales permiten que el elemento con el que concuerdan permanezca no expreso $u$ omitido. Los clíticos verbales son una de las unidades que tienden a alcanzar los mayores niveles de prominencia en el discurso (Ariel 1990 2001) y su capacidad de indexar objetos mediante la concordancia es una de sus principales características (García-Miguel 2015, p. 207). Esto permite establecer la existencia de un sistema de conjugación objetiva en la lengua española (Llorente y Mondéjar, 1974). El clítico de segunda persona te, como morfema de concordancia, resulta suficiente para indexar a la segunda persona, por lo tanto, la expresión del objeto a ti no es necesaria (Aijón Oliva 2017 pp. 3-4; Serrano 2019).

En el ejemplo (1) se observa que la expresión del objeto de persona a ti es innecesaria, ya que el clítico te indexa a la segunda persona.

\section{(1) A ti te llamó tu hermano}

En cuanto al clítico se, este establece la concordancia con el sujeto, lo cual permite la coincidencia del sujeto y del objeto cuando la estructura es reflexiva. 
(2) Juan se lavó

El valor prototípico del clítico se es el reflexivo, sin embargo, la concordancia con el sujeto puede difuminarse o incluso eliminarse, y con ello da lugar a otros valores ya alejados de dicho prototipo, como el pasivo-reflejo o el impersonal (Gómez Torrego 1992 Hernández Sacristán 1997 Monge 2002 [1955]; Mendikoetxea 1999 Sánchez López 2002 entre otros). Cuando el sujeto no es el origen sino el término de la acción, la construcción se convierte en pasiva refleja ("Se venden pisos"). En el caso de las estructuras impersonales con se, no se produce indicación de origen o término de la acción ("Se vive bien en Madrid”). Por todo ello, se ha considerado al clítico se como un marcador de impersonalidad (Sánchez López 2016, pp. 2-3), o como un componente clausal que dota a la cláusula de un valor impersonal (Gómez Torrego 1992, pp. 25-31). Sin embargo, estos distintos valores de se (reflexivo, pasivo-reflejo e impersonal, entre otros apuntados por la tradición) tienen en común la misma base cognitiva: el sujeto se convierte gradualmente en el origen o término de la acción o proceso descrito por el verbo (Aijón Oliva 2010), lo cual permite entender que, en realidad, solo existe un tipo de se que involucra a un referente de forma gradual (Monge 2002 [1955] García 2003).

En esta investigación, las aparentes distintas funciones del clítico se no serán tenidas en cuenta, ya que el estudio se centrará en la forma en la que los distintos significados de las formas te y se construyen distintos mensajes publicitarios. La hipótesis de partida es que, en virtud de la prominencia cognitiva, cada variante (te o se) indexará a un potencial consumidor de forma diferente y tenderá a construir estilos comunicativos diferentes basados en la mayor o menor desubjetivización.

\subsection{El discurso publicitario}

No cabe duda de que el publicitario puede ser considerado como un tipo de discurso concreto (Coleman 1990 Cook 2001 pp. 3-4; Dyer 1982), donde el uso de ciertas estructuras gramaticales puede considerarse recurrente. Este discurso se ha estudiado desde distintos ángulos teóricos, como la Psicología o la Sociología (Danesi 2015, p. 3).

La publicidad trata de alcanzar una comunicación directa y personal con sus potenciales destinatarios. La técnica de la persuasión publicitaria aconseja "haced como si hablarais a vuestro mejor amigo" (Peña Marín 1989, p. 59); sin embargo, en un tipo de comunicación social en la que no existe relación 
personal entre los interlocutores como es la publicitaria, el anunciante ha de construir un modelo de destinatario en función del cual elaborar sus mensajes.

De hecho, en el discurso publicitario los interlocutores pueden considerarse como participantes in absentia (Calsamiglia y Tusón 1999, p. 75). En cualquier caso, si el anunciante pretende de alguna forma generar empatía, ha de transmitir la sensación de tener en cuenta a su interlocutor del mismo modo que lo haría en la conversación cotidiana cara a cara. De ahí que el uso de las posibilidades referenciales que ofrecen las personas gramaticales constituya un recurso muy habitual en el discurso publicitario. Como es lógico, la variabilidad en la referencia suple de alguna manera la ausencia física del interlocutor, a quien conviene tener presente dado su papel destacado en este tipo de interacción, puesto que es a quien se intenta persuadir para que termine realizando la adquisición del producto o la adhesión a la idea publicitada. Además, la publicidad intenta llegar al mayor número de consumidores, de ahí que resulte necesario la alusión al destinatario anónimo, masivo y heterogéneo que caracteriza a los medios de comunicación.

No obstante, el concepto de interlocutor o audiencia en el discurso publicitario es complejo y multidimensional; la audiencia es desconocida, difusa e indefinida, pero el oyente o lector del mensaje suele decodificarse como un destinatario singular (De Cock 2014 p. 24; Goddard 1998 p. 8). De esta forma se satisface el anhelo de acercamiento a un destinatario con el que se simula una conversación de tú a tú, algo que se intenta conseguir mediante el uso predominante de la segunda persona del singular en este tipo de texto. Por ello, si bien es cierto que esta persona gramatical es la más frecuentemente utilizada en el discurso publicitario, en esta investigación se estudiará el uso de esta segunda persona en función de objeto te y la del clítico se, ambas consideradas como recursos de significado que contribuyen a la función comunicativa de la persuasión, presente en el discurso publicitario.

\section{Metodología y corpus}

Para esta investigación se utilizarán textos del Corpus Interaccional del Español, un corpus de español contemporáneo que contiene distintos subcorpus con textos procedentes de distintas situaciones comunicativas: textos conversacionales (79 544 palabras), programas de televisión autonómica (171 258 palabras), redes sociales (13 185 palabras) y textos publicitarios (26 554 palabras). Esta última sección del corpus es la que se utilizará para la presente investigación, la cual consta de la transliteración de 475 anuncios de publicidad obtenidos en 
distintos medios orales y escritos: televisión, prensa y redes sociales. De ellos se obtuvo el total de 824 casos de los clíticos te y se.

El análisis se realizará desde una perspectiva cualitativa y cuantitativa; además del estudio de las propiedades cognitivas de las variantes y el significado que adquieren en los textos publicitarios, se estudiará su distribución sociosituacional mediante un análisis cuantitativo realizado con el paquete estadístico Rbrul (Johnson 2009). Este modelo estadístico analiza la variación como el resultado de la interacción entre factores fijos y aleatorios, lo cual constituye una novedad con respecto a modelos de regresión anteriores como las distintas versiones de GoldVarb o Varbrul. Mediante los porcentajes y pesos que arroja el programa, es posible conocer la significatividad de los factores que covarían con las variantes que se van a estudiar. El valor del peso en los modelos de regresión múltiple, como lo es el modelo Rbrul, indica la frecuencia estadística de una variante en oposición a otra. De forma general, los pesos situados por encima de la cifra de 0.50 se consideran como significativos o favorecedores de una variante, mientras que los que están por debajo de esa cifra se consideran como no significativos.

La relación entre los elementos covariantes puede no ser independiente (Johnson 2013, pp. 302-303), por lo tanto, el modelo de efectos mixtos prevé también la posibilidad de analizar variables continuas y factores concretos considerados como aleatorios (Gorman y Johnson 2013, p. 226; Tagliamonte y Baayen, 2012). Esto constituye una ventaja, puesto que el modelo mixto de regresión solo otorgará significación a un factor si su efecto es más fuerte que el ejercido por otros factores agrupados (Johnson 2009, p. 365).

Se tendrán en cuenta los siguientes factores que covarían con las variantes te y se:

a) La naturaleza oral o escrita del anuncio. Los mensajes publicitarios orales pertenecen a los emitidos por televisión y también en algunas redes sociales como YouTube, mientras que los escritos proceden de publicaciones periódicas (revistas, periódicos) y de redes sociales (Facebook).

b) El consumidor al que va dirigido el anuncio. Se han establecido cuatro categorías: hombre, mujer, jóvenes/niños y familias. Esta división se ha realizado en función de la observación del posible perfil del destinatario o destinatarios del producto anunciado.

c) El grupo de edad al que va dirigido el anuncio. Se consideraron tres categorías: jóvenes, adultos y mayores como posibles grupos de edad de consumidores a los que va dirigido el anuncio.

Para el análisis cuantitativo estos factores se tuvieron en cuenta como factores fijos. Al mismo tiempo, se trató el factor anuncio como aleatorio para 
poder establecer adecuadamente los niveles de interacción y variabilidad entre ellos. No obstante, consideramos que, si bien estudio estadístico de las variantes puede proporcionar interesantes datos acerca del alcance de la variación, en esta investigación esos resultados se combinarán con los que se obtengan del estudio cualitativo y, en cierta medida, estarán supeditados a ellos. Como bien señala Tagliamonte (2011, p. 157), lo importante en los estudios de variación es la dirección explicativa que se proporcione a los datos obtenidos mediante los procedimientos cuantitativos.

\section{Variación de te y se en el discurso publicitario}

De una primera cuantificación general se obtiene, como era de esperar, que la variante te es claramente más frecuente que la variante se en el discurso publicitario (Tabla 1). Es sabido que, de forma general, la segunda persona del singular es la forma más frecuentemente usada, ya que la indexación del interlocutor o audiencia con esta forma constituye una estrategia de persuasión que da a entender que el consumidor es una persona única. A pesar de que los referentes singulares suelen coincidir con su referencia real (Orilia 2009, pp. 12-13), en la publicidad esta singularidad se presenta como una construcción, alejada de la realidad de la audiencia, la cual es por naturaleza difusa e indefinida.

Tabla 1: Distribución del uso de te y se.

\begin{tabular}{lrrrrrr}
\hline & \multicolumn{1}{c}{ Te } & & & \multicolumn{2}{c}{ Se } & Total \\
\cline { 5 - 6 } Casos & $\%$ & & Casos & \% & & \\
\hline 515 & 62,5 & & 309 & 37,5 & 824 \\
\hline
\end{tabular}

En los siguientes ejemplos se aprecia el frecuente uso de la segunda persona en función de objeto te. Con ello el contenido del mensaje se dirige a un interlocutor singular e imita las conversaciones cotidianas cara a cara, donde un potencial hablante se dirige a otro para realizar indicaciones y recomendaciones: "se te hinchan las piernas...te acercas a los días...te notas más hinchada” (1), o "te ayudará...no puedas resistirte" (2).

(1) Se te hinchan las piernas al final del día porque trabajas mucho tiempo de pie. ¿Trabajas en una oficina de farmacia, o en comercio, o en hostelería? 
Cuando hace mucho calor en verano o en invierno con la calefacción, tus piernas se hinchan tanto que hasta duelen. Estás mucho tiempo sentada, sin moverte y tienes calambres e hinchazón en las piernas. Cuando haces viajes largos en avión, tren... se te hinchan tanto los pies que no te entran en los zapatos. Tus piernas están más hinchadas porque has aumentado de peso y no haces mucho ejercicio físico. Cuando te acercas a los días de la regla te notas más hinchada, con la sensación generalizada de haber engordado (CCEC < Adv17-109)

(2) Compensa y disfruta. Nuevo Panrico... Te ayudará a mantener una dieta equilibrada, incluso los días en que no puedas resistirte a tus caprichos, porque solo contiene aquellos azúcares naturalmente presentes en la harina. (CCEC < Adv17-46)

Por su parte, la variante se, aunque menos frecuente que te, se encuentra también de forma regular en los mensajes publicitarios. Si bien la alusión a la segunda persona sigue estando presente en forma de sujeto o de objeto, se puede observar la presencia de se en determinadas cláusulas de los anuncios, lo cual promueve un significado adicional basado, como hemos señalado, en la menor prominencia de esta variante. Así, en el ejemplo (3), se indexa al consumidor ¿Y tú...? y a continuación la frase que le sigue constituye una recomendación realizada con el clítico se "Hay que cuidarse...", además de la estructura declarativa "...ocho de cada diez consumidores se sienten mejor".

(3) Y tú, ¿por qué tomas Vivesoy? "Hay que empezar a cuidarse”. Sandra Belmar. ocho de cada diez consumidores se sienten mejor. Lo que vives hoy, lo disfrutarás mañana (CCEC $<$ Adv17-96)

En (4) alternan las variantes te y se en el mismo enunciado: "te sientes bien y quieres que tu imagen se corresponda...”; resulta evidente que la primera señala al interlocutor imaginario consumidor del producto y la segunda a un elemento léxico de tercera persona se, que concuerda con imagen.

(4) La mejor edad, la tuya. Estás en la mejor edad, y lo sabes. Te sientes bien, y quieres que tu imagen se corresponda con tu autoestima (CCEC < Adv17121)

En el siguiente anuncio, en cambio, la segunda persona aparece más o menos difuminada mediante el posesivo "su" (su juventud), pero este elemento 
léxico queda resaltado como protagonista del mensaje mediante la concordancia que efectúa con el clítico se: "su juventud se derrita al sol".

(5) No deje que su juventud se derrita al sol. Caudalie (CCEC $<$ Adv17-158)

Si bien la variante de objeto te designa claramente al potencial consumidor del producto, la variante se proporciona un significado que desvía la referencia de esa segunda persona y la traslada hacia la esfera nocional de la no persona, lo cual puede apoyar la estrategia persuasiva de los mensajes publicitarios en un sentido claramente distinto al de la segunda persona. Aquellos mensajes con una mayor frecuencia de la variante te estarán orientados hacia la construcción de la subjetivación, mientras que aquellos con se lo harán en un sentido claramente menos subjetivador y, por lo tanto, de alcance general o universal. Los anuncios que incluyen ambas variantes conseguirán dicho efecto de forma combinada, en función de los enunciados donde aparezca cada una de ellas.

Para comprobar esta hipótesis, una primera cuantificación según la naturaleza oral o escrita de los mensajes publicitarios del corpus muestra una cierta inclinación hacia el uso de te en los orales y de se en los escritos (Tabla 2).

Tabla 2: Porcentajes y pesos de las variantes te y se.

\begin{tabular}{lllllll}
\hline & \multicolumn{2}{c}{ Te } & & \multicolumn{2}{c}{ Se } & Total \\
\cline { 2 - 3 } \cline { 5 - 6 } & Casos \% & Pesos & & Casos \% & Pesos & \\
\cline { 2 - 5 } Oral & $150(68,1)$ & 0.579 & & $70(31,9)$ & 0.421 & 220 \\
Escrito & $365(60,4)$ & 0.421 & & $239(39,6)$ & 0.579 & 604 \\
\hline
\end{tabular}

(Anuncio como elemento aleatorio) Random st dev: 1.079; Te: centered factor weight: 0.625 ; Se: centered factor weight: 0.375 .

Estos resultados eran esperables si tenemos en cuenta que la segunda persona, que indexa al interlocutor de forma clara y definida, es la variante que mejor representa las conversaciones cotidianas cara a cara. En (6) y (7) los anuncios se construyen lingüísticamente como si se tratara de una interacción real. En (8), además, se introduce una pregunta retórica que supuestamente va dirigida a un interlocutor singular. Esta construcción del texto es muy frecuente en la publicidad oral: se emplea la segunda persona del singular, acompañada de exclamaciones o preguntas retóricas ¿sabes qué es más grande...?, con ello se logra la cercanía emocional con el potencial consumidor. 
(6) ¡La pasta sabe genial y te deja los dientes súper limpios, a por ellos! (CCEC $<$ Adv17-440)

(7) En Knorr creemos que todos tus platos merecen el mejor sabor por eso Knorr te ofrece una variedad de caldos para hacer que todas tus recetas estén tan sabrosas que incluso necesitarás una mesa más grande (CCEC $<$ Adv17-453)

(8) La Navidad te conecta con los tuyos y eso es muy grande, pero ¿sabes qué es más grande, enorme, estratosférico?, seguir conectados después, esta Navidad pásate a Movistar y contrata fusión contigo (CCEC < Adv17-460)

Por la misma razón, no es inusual que esta variante se encuentre también en los mensajes escritos, sobre todo en aquellos donde se desee dar algún consejo de tipo emocional o personal, como en (9).

(9) Pero ¿sabes qué? Si las personas que te quieren no saben cómo te sientes, tampoco van a poder ayudarte. Y si no pides ayuda, tampoco podrán hacerlo. Aprende a pedir. Posiblemente, te han enseñado a no pedir nada. A que tienes que ser autosuficiente (CCEC $<$ Adv17-616)

Sin embargo, en los mensajes escritos hay mayor presencia de la forma se; dada la inexistencia de una oralidad que pueda simular una interacción concreta, el significado no deíctico de esta variante resulta muy apropiado para describir propiedades generales de algún elemento concreto del enunciado, lo cual proporciona una adecuada estrategia persuasiva. En (10), el infinitivo vestir y el verbo conjugado preocupa acompañados del clítico se, concuerdan con macho, de modo que la persuasión está basada en un hecho que se presenta como una regla general o universal: 'los machos se visten bien'y 'los machos se preocupan por vestirse bien' y en el que cualquier consumidor podría verse reflejado como parte de un conjunto.

(10) Macho es el que se preocupa por vestirse bien. Coca-Cola (CCEC < Adv17-635)

En el siguiente ejemplo (11), la variante se es utilizada para indicar el efecto de una emoción 'nuestra mirada se descoordina'...'se perturban'. Nuevamente, la ausencia de deixis de persona sirve para representar una idea de alcance general, donde el lector puede verse claramente involucrado en el conjunto de esa universalidad. 
(11) Piensa que cuando queremos camuflar una emoción nuestra mirada se descoordina y nuestros músculos oculares se perturban (CCEC < Adv17-721)

De este modo, se puede atisbar que las frecuencias de una y otra variante en los mensajes publicitarios determinarán el significado global del mensaje; los orales tienden al uso de te por su tendencia a la recreación de la interacción cotidiana, mientras que se tiende a aparecer en los mensajes cuyo contenido pretende alcanzar la universalidad de los beneficios del producto, o trata de proyectarse sobre un conjunto amplio de consumidores. Sin embargo, conviene recordar que la segunda persona te es la forma más frecuente (Tabla 1).

Por lo tanto, te y se constituyen dos opciones opuestas en el discurso publicitario: la subjetivación que proporciona la variante te frente a la desubjetivización que aporta la variante se. Aun así, es posible que ambas variantes coaparezcan en un mismo anuncio, en cuyo caso cada enunciado adquirirá el significado correspondiente. Por ejemplo, en (12) se parte de un enunciado de contenido generalizador '...se confiesan...' para pasar luego a una indexación directa al interlocutor imaginario 'te las contamos'.

(12) ¿Por qué algunas personas que se confiesan enamoradas son infieles? Las razones pueden ser varias y te las contamos en el siguiente artículo (CCEC $<$ Adv17-739)

La distribución de cada variante en los distintos textos publicitarios servirá también para orientar la estrategia persuasiva en función de la mayor o menor personalidad proporcionada por cada variante, que será más o menos útil en función del consumidor destinatario del anuncio. Por ello, para profundizar en esta distribución, se realizó una cuantificación de la aparición de cada forma según el destinatario del mensaje. Según se observa en la Tabla 3, la variante te es la que alcanzó pesos de mayor significatividad en todas las categorías de destinatario, especialmente entre la de las mujeres, seguida de las de jóvenes y niños, familias y, por último, de la de hombres. Sin embargo, debido a la escasez de ejemplos obtenidos en la categoría de hombres y de jóvenes y niños, centraremos el análisis cualitativo en torno a los grupos de mujeres y familias.

Por lo que respecta a se, si bien la estadística no concede significatividad a ninguna categoría de los consumidores destinatarios, cabe señalar un ligero incremento del peso estadístico entre la categoría hombres.

Como es sabido, actualmente una gran cantidad de anuncios están destinados a la mujer, básicamente para fomentar el consumo de productos de belleza. De hecho, de 475 anuncios analizados, 201 pertenecen a anuncios de este sector. Como bien se refleja en la Tabla 4, el $90 \%$ de ellos tienen como destinatario del 
Tabla 3: Porcentajes y pesos de las variantes te y se según el tipo de consumidor destinatario del producto anunciado.

\begin{tabular}{lrrrrrr}
\hline & \multicolumn{2}{c}{$T$ Te } & & \multicolumn{2}{c}{ Se } & Total \\
\cline { 2 - 3 } & Casos y porcentajes & Peso & & Casos y porcentajes & Peso & \\
\cline { 2 - 3 } Mujeres & $201(66,1 \%)$ & 0.661 & & $103(33,9 \%)$ & 0.339 & 304 \\
Hombres & $15(51,7 \%)$ & 0.517 & & $14(48,3 \%)$ & 0.483 & 29 \\
Jóvenes y niños & $26(62 \%)$ & 0.619 & & $16(38 \%)$ & 0.381 & 42 \\
Familias & $(60,8 \%)$ & 0.608 & & $176(39,2 \%)$ & 0.392 & 449 \\
\hline
\end{tabular}

(Anuncio como elemento aleatorio) Random st.dev: 1.032; Te: centered factor weight: 0.625; Se: centered factor weight: 0.375 .

Tabla 4: Porcentajes de distribución de los destinatarios de los productos de belleza.

\begin{tabular}{rrrrrr}
\hline & Mujeres & Familias & Jóvenes & Hombres & Total \\
\hline Belleza & $181(90 \%)$ & $11(5,4 \%)$ & $7(3,5 \%)$ & $2(0,1 \%)$ & 201 \\
\hline
\end{tabular}

producto al género femenino. De hecho, dada su presencia sustancial en el discurso publicitario, la categoría belleza o cosmética ha sido considerada como un subgénero de este tipo de discurso (Ringrow 2016, p. 18).

Así pues, podemos explicar la tendencia de la variante te a aparecer en los mensajes publicitarios destinados a la mujer, y a la vista de los resultados cuantitativos, podemos explicar también que con gran frecuencia estos pertenezcan al sector publicitario de la belleza. Resulta lógico que se emplee la variante personal (te), que señala directamente al interlocutor (en este caso la mujer) como destinatario de los beneficios del producto anunciado. El uso de la segunda persona en la publicidad de productos de belleza femenina ha sido considerado como una estrategia para dar la impresión de que el producto ha sido creado específicamente para la mujer y que lo necesita (Ringrow 2016, p. 50), pero, sobre todo, que es merecedora del mismo. Véanse los ejemplos (13)-(15).

(13) Siéntete aún más guapa cada día. ¡Te lo mereces! Bodybell. Sonríe porque ahora estar aún más guapa te costará muy poco $($ CCEC $<$ Adv17-68)

(14) El ritmo intenso del día hace que tengamos muy poco tiempo para nosotras. Por ello, Clinique te propone un maquillaje perfecto en tan solo 90 segundos $($ CCEC $<$ Adv17-204) 
(15) ¿Qué color prefieres para tus ojos? Te presentamos las nuevas sombras de ojos en crema de MAKE UP FOR EVER. 22 colores intensos, cremosos, irisados, wáterproof, muy fáciles de aplicar para un efecto espectacular. Entenderás por qué MAKE UP FOR EVER es la marca más utilizada por los profesionales. Sephora (CCEC $<$ Adv17-42)

Aunque cada vez viene siendo más frecuente la promoción de productos de belleza destinados a los hombres, en este corpus solo hemos obtenido dos ejemplos de anuncios de esta categoría. La construcción de las categorías hombre y mujer en el discurso publicitario está fuertemente marcada (Coupland 2007, p. 41); los anuncios destinados a la mujer suelen recrearse en la consecución o preservación directa de la belleza y cuidados femeninos, mientras que los Dedicados a los hombres normalmente utilizan recursos y técnicas menos directas y más metafóricas (Coupland 2007, pp. 53-54). Como norma general, se observa una distinción entre ambos tipos de texto y se suelen fomentar aspectos estereotipados de cada sexo/género (Coupland 2003). En este sentido, en este trabajo hemos podido confirmar en cierta medida que el consumidor femenino o masculino del anuncio promueve el uso diferenciado de las variantes te y se.

La cercanía nocional que proporciona la variante te frente a otras posibilidades resulta especialmente apta también para el grupo de consumidores familia, incluso cuando la audiencia se supone plural, ya que el producto anunciado lo podrá consumir o disfrutar cualquier miembro de la familia, como se observa en los ejemplos (16)-(18).

(16) Anticípate al frío, instala ahora gas natural y ahorra 200 euros (CCEC $<$ Adv17-647)

(17) Si crees que has tocado fondo y tienes un perro, no te queda una esperanza, te queda la mejor (CCEC $<$ Adv17-751)

(18) Estas Navidades esperemos que todos te ayudemos a hacer la cena $($ CCEC $<$ Adv17-549)

En el ejemplo (19) un diálogo entre niños sirve para promocionar un producto de alimentación infantil donde nuevamente se representa ficticiamente un diálogo que podría haberse producido en la vida cotidiana.

(19) A: ¿Qué haces? es que me voy de excursión y quiero llevar mi ColaCao. $\mathrm{B}:$ ¡Ay enano, que no te enteras! el Cola Cao que se lleva es este (CCEC $<$ Adv17-533) 
En definitiva, la variante te aparece en los anuncios que construyen un significado deíctico y personalizado, en definitiva, subjetivador, que indexa al interlocutor para hacerle partícipe en el mismo e involucrarlo en el pretendido consumo del producto. En general, en todas las categorías de productos anunciados predomina el uso de te, no obstante, se observa un incremento en aquellos destinatarios que requieren una mayor personalización, como la categoría mujeres. Los anuncios en los que ellas son las posibles consumidoras (sobre todo en aquellos de productos de belleza) se construyen con la variante más prominente te, que da lugar a un significado claramente subjetivador.

Contrariamente, el ligero incremento de la presencia de se en los anuncios destinados a los hombres se explica también por el menor nivel de prominencia cognitiva de esta forma frente a te, que, contrariamente, crea un significado desubjetivizador. De la misma forma que se aprecia en el ejemplo (10), en el ejemplo (20) la variante se concuerda con algún elemento léxico de tercera persona: 'el niño que fuiste'. Con ello se evita focalizar sobre el consumidor en forma de segunda persona, como ocurriría con la variante te.

(20) Que el niño que fuiste no se avergüence del adulto que eres (CCEC $<$ Adv17-241)

Asimismo, en el ejemplo (21), la segunda persona está ausente y en su lugar aparece la primera del plural como posesivo nuestra y la concordancia con un elemento no personal "eso se nota".

(21) En cada Citroen C4 va una parte nuestra y eso se nota (CCEC < Adv17-363)

En estos mensajes publicitarios destinados a un consumidor masculino se crea un significado desubjetivizador. De esta cuantificación se obtienen datos interesantes sobre la publicidad destinada a la mujer y al hombre y sobre la construcción de los modelos de sexo/género en la sociedad. La publicidad de la belleza y la cosmética es una industria culturalmente extendida donde la mujer es la gran protagonista del posible consumo. La publicidad tiende a construir un modelo de feminidad basado en la belleza y en la sensualidad, en la solución de problemas de belleza que supuestamente tienen todas las mujeres y que desean solucionar (Ringrow 2016, pp. 59-60). Por este motivo, la indexación del referente de segunda persona en este tipo de mensaje publicitario resulta fundamental, y esa puede ser una de las razones de la menor incidencia del uso de la variante te en la publicidad destinada al género masculino.

Para avanzar en el conocimiento del uso de estas variantes en el discurso publicitario, resulta útil conocer su distribución entre los distintos grupos de 
Tabla 5: Porcentajes y pesos de las variantes te y se según la edad del tipo de consumidor destinatario del producto anunciado.

\begin{tabular}{|c|c|c|c|c|c|}
\hline & \multicolumn{2}{|c|}{$T e$} & \multicolumn{2}{|c|}{ Se } & \multirow{2}{*}{ Total } \\
\hline & Casos $\%$ & Peso & Casos \% & Peso & \\
\hline Jóvenes & $29(46,1)$ & 0.460 & $34(53,9)$ & 0.540 & 63 \\
\hline Adultos & $475(64,1)$ & 0.640 & $267(35,9)$ & 0.360 & 742 \\
\hline Mayores & $11(57,9)$ & 0.579 & $8(42,1)$ & 0.421 & 19 \\
\hline
\end{tabular}

(Anuncio como elemento aleatorio) Random st.dv: 1.01; Te: centered factor weight: 0.625; Se: centered factor weight: 0.375 .

edad de los potenciales consumidores (Tabla 5). Los porcentajes y los pesos indican claramente que la variante te está más presente en los anuncios dedicados a los adultos seguidos de los mayores, mientras que se lo hace en aquellos dedicados a los consumidores de menor edad. Sin embargo, a juzgar por el escaso número de casos obtenido en la categoría de mayores (11) y el elevado en el de adultos (475), solo se deberían tener en cuenta los resultados de este último grupo.

Por lo que respecta al grupo de menor edad (jóvenes), que alcanza un peso significativo en la variante $s e$, es necesario señalar que una tabulación cruzada con el grupo de destinatarios del anuncio indica que, si bien el mayor número de casos se muestra en el grupo de jóvenes y niños $(60,3 \%)$, el $30,1 \%$ se corresponde con el destinatario mujeres, que como se ha visto, es junto con las familias, el grupo de potencial consumidor más frecuente de los mensajes publicitarios (Véase Tabla 3). Como se observa en la Tabla 6, un tercio de los casos de ambas variantes está dirigido a la categoría mujeres jóvenes.

Tabla 6: Tabulación cruzada de las categorías de destinatarios de los mensajes publicitarios.

\begin{tabular}{lrrrrr}
\hline & Mujeres & Hombres & Jóvenes y niños & Familias & Total \\
\hline Jóvenes & $19(30,1 \%)$ & $5(8 \%)$ & $38(60,3 \%)$ & $1(1,6 \%)$ & 63 \\
Adultos & $273(36,8 \%)$ & $24(3,2 \%)$ & 0 & $445(60 \%)$ & 742 \\
Mayores & $16(84,2 \%)$ & 0 & 0 & $3(15,8 \%)$ & 19 \\
\hline
\end{tabular}

Aunque pueda parecer lo contrario, no existe solapamiento entre los factores jóvenes y niños y jóvenes. El primer factor se integra en la categoría "grupo de consumidor" en la cual puede existir un espectro amplio de edades y el segundo se refiere estrictamente a la categoría edad y contrasta con los factores adultos y mayores. 
La variante subjetivadora (te) es más frecuentemente empleada en mensajes dirigidos a un consumidor adulto, como (22), que contiene un consejo de tipo emocional, o en (23), donde se promociona un establecimiento de alimentación.

(22) Suena el despertador. Abres los ojos con ilusión. Hoy será un buen día, lo presientes. Tienes cantidad de planes. Te levantas de un salto. Abres la ventana y miras hacia afuera. Hay un sol radiante. La luz es espectacular. ¿Cómo te sientes? (CCEC < Adv17-625)

(23) Todo lo que necesitas cuando lo necesitas. Alimentación y bebidas. Perfumería y droguería. Ocio y regalos. Sólo Opencor te sienta así de bien. Porque sólo en OpenCor compras lo que te gusta, como a ti te gusta $($ CCEC < Adv17-363)

También es más frecuente el empleo de te para los destinatarios mayores, si bien, como se desprende de la tabulación cruzada (Tabla 5), estos son en su mayoría mujeres, aunque la escasez de ejemplos no permite extraer conclusiones demasiado contundentes.

(24) Para verse joven a cualquier edad, no dejes que te lo cuenten $($ CCEC $<$ Adv17-575)

De nuevo, son las mujeres y los anuncios dedicados a la belleza femenina los que obtienen más representatividad en la categoría de adultos. De hecho, el anuncio de productos de belleza y el destinatario mujeres recubren la mayor parte de la publicidad oral y escrita en la actualidad.

(25) Dime qué color te gusta y te diré como eres (CCEC < Adv17-225)

(26) Te estamos buscando a ti ¿eres mujer? ¿quieres disfrutar de una buena piel? Si es así, entonces tienes todo lo necesario para ser una de las cuatro protagonistas de la experiencia Clinique (CCEC $<$ Adv17-208)

Como se desprende de los resultados de la Tabla 4, los mensajes destinados a los jóvenes muestran un peso significativo de la variante se, aunque una parte de ellos, como se señaló, forman parte de la categoría mujeres. El ejemplo (27) se construye básicamente en tercera persona, con una elevada presencia de la forma se. Esta construcción pretende llegar a un grupo 
de jóvenes en un conjunto de acciones que se presentan como estereotipadas en los que se pueden sentir incluidos. La estrategia persuasiva está centrada precisamente en el valor desubjetivizador que proporciona la variante se. Solo el último enunciado se formula con una segunda persona del plural les.

(27) Bueno, a ver, que levante la mano al que alguna vez dejaron plantado en un bar [...] el que se enganchó con una telenovela [...] el que se fue de casa de mamá a los 35 [...] la que renegó de la madre y terminó pareciéndose a ella [...] los que cantan en inglés, por fonética [...] el que se va a casar y tiene miedito [...] el que se quedaba calvo y se rapó para ser sexy. Y ahora sí les pido que levanten la mano los que dijeron yo nunca voy a tomar Coca-Cola light y ahora la piden en cualquier bar como si nada $($ CCEC $<$ Adv17-93)

Asimismo, el uso de se en los anuncios para este rango de edad sirve para representar instrucciones de uso o recomendaciones de alcance general, sobre todo en los de juguetes o elementos de diversión, si bien normalmente aparece en alternancia con la segunda persona. El primer enunciado en (28) describe las características del personaje Mickey para pasar luego a la indexación directa del consumidor, mientras que en (29) la indicación 'se mueve como una gatita' aparece después de la descripción del juguete, que incluye una alusión a la segunda persona 'si le acaricias...'.

(28) Mickey sabe cómo divertirse y mantenerse activo con sus amigos, ¿te gustaría jugar con ellos? (CCEC < Adv17-697)

(29) Zoomer Kitty, si le acaricias la mejilla, Kitty ronronea de verdad, es tan adorable, se mueve como una gatita y le encanta saltar y seguir mi mano (CCEC $<$ Adv17-699)

En el ejemplo (30), un anuncio de automóviles con caricaturización animada utiliza la forma se. En él se representan también ideas generales y universales del producto anunciado mediante el empleo de la variante se.

(30) En un mundo separado, una valiente muñeca y un intrépido coche se unen con un objetivo: cambiar las reglas del juego, esta Navidad Audi presenta: la muñeca que eligió conducir, una historia que empieza cuando las luces se apagan (CCEC < Adv17-712) 


\section{La variación de te y se como creación de estilos comunicativos en el discurso publicitario}

Como se ha indicado, la mayor prominencia cognitiva de la variante te permite la indexación concreta del referente de segunda persona en el discurso publicitario, que, aun tratándose de una audiencia difusa e indefinida, individualiza casi categóricamente a dicho referente para que se sienta único y singular como consumidor. Se ha demostrado que cada producto puede adoptar un estilo comunicativo propio como estrategia persuasiva en el ámbito publicitario (Danesi 2015, pp. 8-9). La construcción de los mensajes con la forma de objeto te proporciona un significado de subjetivación que no posee la forma se, la cual, debido a su menor prominencia, crea un significado basado en la desubjetivización del contenido. El uso de una de las formas o la alternancia de ambas crean estilos comunicativos en los textos publicitarios. Dichos estilos, basados en propiedades cognitivas, pueden observarse de forma regular en función del tipo de destinatario del mensaje.

Así pues, se puede observar que los anuncios emitidos en canales orales, los destinados a las mujeres y a la categoría de adultos tienden a construirse con un estilo más subjetivo o subjetivador, ya que permiten insertar idealmente al referente en el mensaje e involucrarlo de forma más específica y persuasiva en el consumo. Esto sucede porque la publicidad está construida básicamente en torno a estas categorías, que podrían considerarse como destinatarios prototípicos en los anuncios publicitarios, no solo en virtud de su cantidad, sino también en función de la rentabilidad que supone la construcción de anuncios para este tipo de consumidor.

Por esa razón, la tendencia más habitual en los mensajes publicitarios es la de construir mensajes donde la persuasión emocional esté basada en la subjetivación, a través del uso de distintos recursos, entre los que se encuentra el uso de la segunda persona en función de sujeto y objeto, como se aprecia en el ejemplo (31).

(31) Generación mamá. Todos los ojos te miran cuando dejas a los niños en el colegio e incluso cargada con las bolsas de la compra, siempre tienes un atractivo especial. No lo dudes, no puedes evitar estar magnífica. Y para que sigas así, Sephora te ha reservado una selección de productos más brillantes que la mejor joya $(\mathrm{CCEC}<\mathrm{Adv17-67)}$

Desde una perspectiva cognitiva como la que se adopta en este trabajo, la subjetividad es el producto de la conceptualización de la realidad desde el punto 
de vista de los sujetos y, consecuentemente, desplazada de los objetos (Croft y Cruse 2004 pp. 91-93; Cuenca y Hilferty 1999 pp. 162-167), o bien la manifestación del punto de vista o actitud del hablante hacia el discurso o el contenido proposicional (Scheibman 2007, p. 122). Cuando la gramática ofrece distintas posibilidades de describir la realidad, la elección del hablante puede ser indicativa de un enfoque orientado cognitivamente hacia la subjetividad (Israeli 1997 p. 14; Scheibman 2001 p. 61). Al mismo tiempo, esto demuestra que las estructuras gramaticales sirven a distintos propósitos comunicativos en el discurso (Englebretson 2007, pp. 105-106). Por tanto, la noción de subjetividad o subjetivación implica al participante en la construcción lingüística (Brems, Ghesquière y Van de Velde, 2014 p. 2; Cornillie y Delbecque, 2006).

La subjetivación, basada en la personalización del destinatario, resulta útil como estrategia persuasiva para los destinatarios mencionados. No obstante, hay que insistir en que el uso de la segunda persona del singular en el discurso publicitario es la variante más frecuentemente usada, por lo que podría tratarse de un prototipo discursivo publicitario (Serrano 2018).

Por el contrario, la frecuencia en el empleo de la variante se, debido a su menor prominencia cognitiva, con la que no se focaliza sobre ningún potencial interlocutor o consumidor, promueve un significado basado en la desubjetivización. Este valor se emplea en el discurso publicitario como una alternativa que representa las bondades del producto como inespecíficas, universales o generalizadoras, y que tiende a aparecer en los mensajes escritos, en los dirigidos a los hombres y a los consumidores de menor edad. Véanse los ejemplos (32) y (33) donde el mensaje publicitario desvía la predicación de una persona gramatical y la centra en consideraciones de carácter universal.

(32) Se corre como se entrena. Nueva temporada otoño invierno 2016 $($ CCEC $<$ Adv17-217)

(33) El otoño se lleva (CCEC < Adv17-224)

Las construcciones lingüísticas desplazadas de los sujetos y basadas en los objetos pueden producir la objetivación discursiva. En este caso, el uso de la variante se, por su orientación referencialmente terciopersonal o no personal conlleva la creación de un estilo menos subjetivo que las variantes personales o tendentes a la desubjetivización (Langacker 1994 pp. 15-16; Lyons 1981 p. 38). En suma, la estrategia persuasiva de los mensajes publicitarios está fundamentada en los valores de significado que proporcionan las variantes y en los estilos comunicativos, más o menos subjetivos, que se crean con ellas. 


\section{Conclusiones}

Esta investigación ha tratado de demostrar que los significados cognitivos que subyacen a las elecciones morfosintácticas variables crean estilos comunicativos en los textos publicitarios. La principal noción cognitiva aplicada para el caso de la variación entre los clíticos te y se ha sido la prominencia cognitiva, una conceptualización que representa la accesibilidad o mantenimiento del referente del objeto de persona ( $a$ ti) sin necesidad de que este se exprese, y que se manifiesta mediante el correspondiente morfema de concordancia te.

Puesto que el discurso publicitario posee una innegable naturaleza persuasiva, el uso de una u otra variante en los distintos mensajes contribuirá a que el anuncio se oriente cognitivamente hacia un estilo más o menos desubjetivizador. Un análisis cuantitativo de la distribución de cada una de las variantes en función de varias características de los anuncios revela que cada una de ellas tiende a aparecer en mensajes de distinta naturaleza. La forma te se utiliza con más frecuencia en los mensajes orales, en los destinados a las mujeres, familias y adultos. Estas categorías son, por otra parte, para las que se crean más mensajes publicitarios, lo cual coincide con el alto empleo de la forma de segunda persona te. En cambio, la variante se, menos frecuente, alcanza mayor frecuencia en los mensajes escritos, entre los hombres y los jóvenes. Todo ello está conectado con la identidad que se le quiera proporcionar al potencial consumidor del producto; si bien la publicidad está claramente enfocada al género femenino, (especialmente en la promoción de productos de belleza), es posible observar cierta construcción de las identidades de los consumidores a partir de la perpetuación de ciertos estereotipos, sobre todo los relativos al sexo/género de estos (Ringrow 2016 Serrano 2019).

Si partimos de la idea de que el discurso publicitario, que representa la función comunicativa de la persuasion, debe ser por naturaleza subjetivo, no es de extrañar que la persona del discurso más presente en el mismo sea la segunda persona. En este trabajo se ha podido corroborar que la forma de objeto te es claramente más frecuente que la del clítico se, y ello porque esta forma, en función de su significado cognitivo, contribuye a la subjetivización del contenido, como se ha señalado. Con todo, la función que cumple la variante se en este tipo de discurso merece ser también tenida en cuenta, ya que constituye un recurso que orienta los contenidos hacia la desubjetivización, lo cual puede ser también muy útil para lograr la persuasión en cierto tipo de consumidores, como se ha demostrado.

En suma, como discurso planificado que es, en la publicidad se puede observar cómo la variación morfosintáctica crea significados que conllevan la 
creación de estilos comunicativos diferenciados que se distribuyen según el distinto tipo de consumidor para lograr la efectividad de la función comunicativa de la persuasion.

\section{Fundref}

Este trabajo forma parte del proyecto "Variación gramatical y creación del significado en español: los objetos centrales y las construcciones desubjetivizadoras”, financiado por el Ministerio de Economía, Industria y Competitividad (FFI2016-74825P) y por el programa "María Rosa Alonso" de ayudas para el fomento de la investigación en Ciencias Sociales e Humanidades, financiado por el Cabildo Insular de Tenerife. Agradezco a Miguel A. Aijón Oliva (Universidad de Salamanca) las observaciones y recomendaciones realizadas al manuscrito.

\section{Referencias bibliográficas}

Aijón Oliva, M. A. (2010). La variación de se en las estructuras pluriverbales:

Variacióny significado. Boletín de Filología de la Universidad de Chile, XLV (2), pp.11-29.

Aijón Oliva, M. A. (2017). Speaker self-profiling through discursive indexation and syntactic encoding in Spanish radio talk. Borealis. An International Journal of Hispanic Linguistics, 6 (1), pp. 175-199.

Aijón Oliva, M. A. (2018). Constructing us. The first and second persons in Spanish media discourse. Berlin: Mouton de Gruyter (in press).

Aijón Oliva, M. A. y Serrano, M. J. (2013). Style in Syntax. Investigating variation in Spanish pronoun subjects. Bern: Peter Lang.

Anderson, S. (2005). Aspects of the theory of clitics. Oxford: Oxford University Press.

Ariel, M. (1990). Accessing noun phrase antecedents. London: Routledge.

Ariel, M. (2001). Accessibility theory: An overview. En Sanders, T. Schilperoord, J. y Spooren, W. (Eds.), Text representation: Linguistic and psycholinguistic aspects (pp. 29-87). Amsterdam: Benjamins.

Brems, L., Ghèsquiere, L. y Van de Velde, F. (2014). Intersections of intersubjectivity. En Brems, L., Ghèsquiere, L. y Van de Velde, F. (Eds.), Intersubjectivity and intersubjectification in grammar and discourse. Theoretical and descriptive Advances (pp. 1-7). Amsterdam: Benjamins.

Calsamiglia, H. y Tusón, A. (1999). Las cosas del decir. Manual de Análisis del Discurso. Barcelona: Ariel.

Coleman, L. (1990). The language of advertising. Journal of Pragmatics, 14 (1), pp. 137-145.

Cook, G. (2001). The Discourse of Advertising. London: Routledge.

Cornillie, B. y Delbecque, N. (Eds.) (2006). Topics in subjectification and modalization. Amsterdam: Benjamins. 
Coupland, J. (2003). Ageist ideology and discourses of control in skincare product marketing. En Coupland, J. y Gwyn, R. (Eds.), Discourse, the body and identity (pp. 127-150). Basingstoke: Palgrave Macmillan.

Coupland, J. (2007). Gendered discourses on the 'problem' of ageing: Consumerised solutions. Discourse and Communication, 1 (1), pp. 37-61.

Croft, W. y Cruse, A. D. (2004). Cognitive Linguistics. Cambridge: Cambridge University Press. Cuenca, M. J. y Hilferty, J. (1999). Introducción a la Lingüística Cognitiva. Barcelona: Ariel.

Danesi, M. (2015). Advertising discourse. En Tracy, K. Ilie, C. y Sandel, T. (Eds.), The international encyclopedia of language and social interaction (pp. 2-10). Oxford: Wiley.

De Cock, B. (2014). Profiling participants in discourse. Form and function in Spanish conversations and debates. Amsterdam: Benjamins.

Dyer, G. (1982). Advertising as communication. London: Routledge.

Englebretson, R. (2007). Stancetaking in Discourse: Subjectivity, Interaction, Evaluation. Amsterdam: Benjamins.

García, E. (2003). Modern Spanish "Spurious” Se: A genuine case of pseudo analysis. Linguistics, 41, pp. 989-1039.

García-Miguel, J. M. (2015). Variable coding and object alignment in Spanish: A corpus-based approach. Folia Linguistica, 49, pp. 205-256.

Ginzburg, J. (2012). Interaction, grammar, and the behavioural sciences. Oxford: University Press.

Goddard, A. (1998). The Language of advertising. London: Routledge.

Goldberg, A. (2005). Constructions at work. The nature of generalization in language. Oxford: University Press.

Gómez Torrego, L. (1992). Valores gramaticales de se. Madrid: Arco Libros.

Gorman, K. y Johnson, D. E. (2013). Quantitative analysis. En Bayley, R. Cameron, R. y Lucas, C. (Eds.), The Oxford handbook of sociolinguistics (pp-215-240). Oxford: University Press.

Gundel, J. K., Hedberg, N. y Zacharski, R. (1993). Cognitive status and the form of referring expressions in discourse. Language, 69, pp. 274-307.

Haspelmath, M. (2013). Argument indexing: A conceptual framework for the syntax of bound person forms. En Bakker, D. y Haspelmath, M. (Eds.), Languages across boundaries: Studies in memory of Anna Siewierska (pp. 197-226). Berlin: Walter de Gruyter.

Hernández Sacristán, C. (1997). Construcciones reflejas. Español Actual, 67, pp. 17-24.

Israeli, A. (1997). Semantics and pragmatics of the "reflexive" verbs in Russian. München: Verlag Otto Sagner.

Johnson, D. E (2009). Getting off the GoldVarb standard: Introducing Rbrul for mixed-effects variable rule analysis. Language and Linguistics Compass, 3 (1), pp. 359-383.

Johnson, D. E. (2013). Descriptive statistics. En Podesva, R. y Sharma, D. (Eds.), Research Methods in Linguistics. Cambridge: University Press, pp. 288-315

Langacker, R. W. (1991). Foundations of Cognitive Grammar, vol. II: Descriptive application. Stanford: Stanford University Press.

Langacker, R. W. (1994). The limits of continuity: Discreteness in cognitive semantics. En Fuch, C. y Victorri, B. (Eds.), Continuity in linguistic semantics (pp. 9-20). Amsterdam: Benjamins.

Langacker, R. W. (2009). Investigations in Cognitive Grammar. Berlin: Mouton de Gruyter.

Llorente, A. y Mondéjar, J. (1974). La conjugación objetiva en español. Revista Española de Lingüística, 4, pp. 1-60.

Lyons, J. (1981). Language and Linguistics. Cambridge: Cambridge University Press. 
Mendikoetxea, A. (1999). Construcciones con se: medias, pasivas e impersonales. En Bosque, I. y Demonte, V. (Eds.), Gramática descriptiva de la lengua española (pp. 1631-1722). Madrid: Espasa Calpe.

Miller, P. y Monachesi, P. (2003). Les pronoms clitiques dans les langues romanes. En Danièle, G. (Ed.), Les langues romanes, problèmes de la phrase simple (pp. 67-123). Paris: CNRS éditions.

Monge, F. (2002 [1955]). Las frases pronominales de sentido impersonal en español. En Sánchez López, C. (Ed.), Las construcciones con se (pp. 341-391). Madrid: Visor Libros.

Orilia, F. (2009). Singular reference: A descriptivist perspective. London: Springer.

Peña Marín, C. (1989). El estribillo de los fantasmas. Revista de Occidente, 92, pp. 57-64.

Ringrow, H. (2016). The language of cosmetic advertising. London: Mcmillan.

Romano, M. y Porto, M. D. (2016). Discourse, cognition and society. En Romano, M. y Porto, M.D. (Eds.), Exploring discourse strategies in social and cognitive interaction (pp.1-20). Amsterdam: Benjamins.

Sánchez López, C. (Ed.) (2002). Las construcciones con se. Madrid: Visor Libros.

Sánchez López, C. (2016). Pasividad e impersonalidad. En Gutiérrez-Rexach, J. (Ed.), Enciclopedia de Lingüística Hispánica (pp. 773-784). Londres: Routledge.

Scheibman, J. (2001). Local patterns of subjectivity in person and verb type in American English Conversation. En Bybee, J. y Hopper, P. (Eds.), Frequency and the emergence of linguistic structure (pp. 61-89). Amsterdam: Benjamins.

Scheibman, J. (2007). Subjective and intersubjective uses of generalizations in English conversation. En Englebretson, R. (Ed.), Stancetaking in discourse subjectivity, interaction, evaluation (pp. 111-138). Amsterdam: John Benjamins.

Serrano, M. J. (2016). La variación sintáctica. En Gutiérrez-Rexach, J. (Ed.), Enciclopedia de Lingüística Hispánica (pp. 809-821). Londres: Routledge.

Serrano, M. J. (2018). Deictic and desubjectivizing meaning in advertising discourse: $T e$ and se clitics. Lingua. An International Review of General Linguistics (in press).

Serrano, M. J. (2019). Los objetos verbales de persona como variantes de tratamiento interpersonal canario en la red social Facebook. Revista Española de Lingüística Aplicada, 32 (1), (en prensa).

Tagliamonte, S. A. (2011). Variationist sociolinguistics. Change, observation, implication. Oxford: Wiley-Blackwell.

Tagliamonte, S. A. y Baayen R. H. (2012). Models, forests and trees of York English: Was/were variation as a case study for statistical practice. Language Variation and Change, 24 (2), pp. 135-178. 